Title:

Author(s):

Submitted to:

\section{Wavelet Theory and Its Applications}

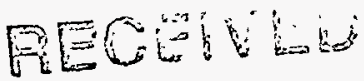 \\ JUㄴ 28 19อง \\ OSTI
}

\section{Vance Faber, CIC-3}

Jonathan Bradley, $\mathrm{ClC}-3$

C. Brislawn, ClC-3

Randy Dougherty, CIC-3

Michael Hawrylycz, CIC

\section{DOE Office of Scientific and Technical} Information (OSTI)

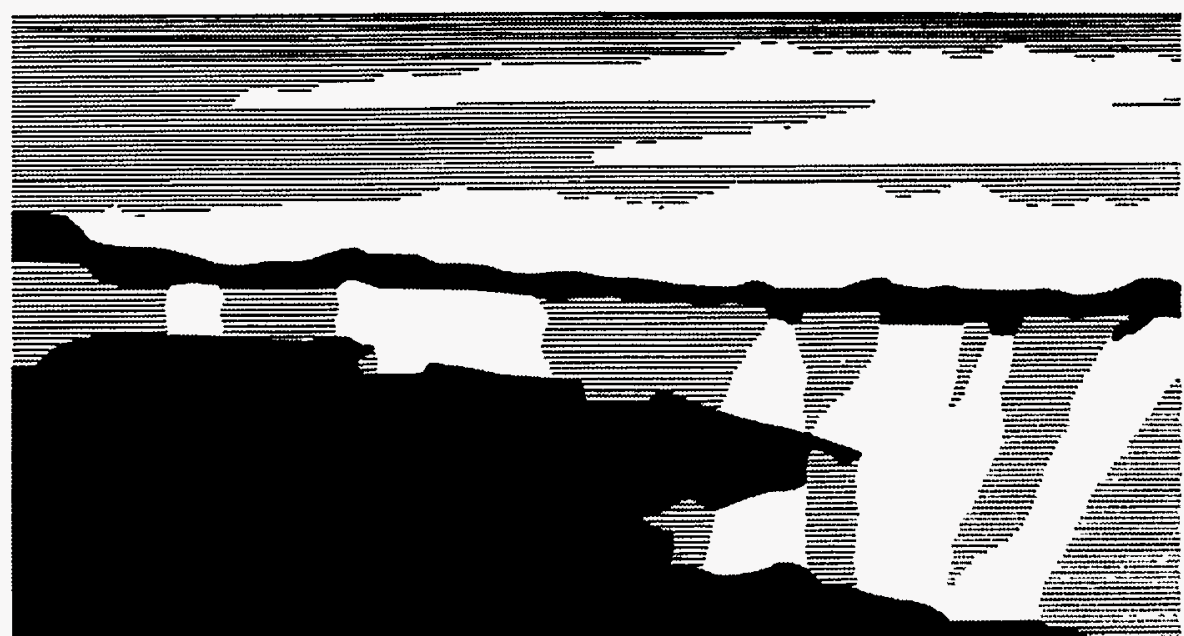

Los Alamos National Laboratory, an affirmative action/equal opportunity employer, is operated by the University of Califomia for the U.S. Department of Energy under contract W-7405-ENG-36. By acceptance of this article, the publisher recognizes that the U.S. Government retains a nonexclusive, royaltyfree llcense to publish or reproduce the published form of this contribution, or to allow others to do so, for U.S. Govemment purposes. The Los Alamos National Laboratory requests that the publisher identify this article as work performed under the ausp "es the 


\title{
Wavelet Theory and Its Applications
}

\author{
Vance Faber*, Jonathan Bradley, C Brislawn, \\ Randy Dougherty, and Michael Hawrylycz
}

\begin{abstract}
This is the final report of a three-year, Laboratory-Directed Research and Development (LDRD) project at the Los Alamos National Laboratory (LANL). We investigated the theory of wavelet transforms and their relation to Laboratory applications. The investigators have had considerable success in the past applying wavelet techniques to the numerical solution of optimal control problems for distributed-parameter systems, nonlinear signal estimation, and compression of digital imagery and multidimensional data. Wavelet theory involves ideas from the fields of harmonic analysis, numerical linear algebra, digital signal processing, approximation theory, and numerical analysis, and the new computational tools arising from wavelet theory are proving to be ideal for many Laboratory applications.
\end{abstract}

\section{Background And Research Objectives}

The theory of wavelet transforms is a recent development drawing contributions from a number of different fields, including harmonic analysis, digital signal processing, approximation theory, and numerical analysis. A wavelet transform provides a linear representation of functions in terms of dilates and translates of a suitable basis function-the mother wavelet. The basis vectors are localized spatially by the translation parameter and lie on exponentially scaled intervals in the frequency domain, so wavelet expansions enjoy simultaneous time/frequency localization that is lacking in Fourier expansions, plus an octavescaled frequency resolution that makes them particularly economical for analyzing processes whose spectrum is biased towards lower frequencies, including much high-resolution sensor data.

Wavelet expansions also exist for digital signals and are known as discrete wavelet transforms (DWTs). Such transforms are instances of multirate digital filter banks having the special property that, in the infinite sampling rate limit, they converge to a decomposition of the corresponding continuous-time signal in terms of a basis of continuous wavelets. This means that there is a duality for wavelet transforms analogous to the relationship between the

\footnotetext{
*Principal investigator, e-mail: vxf@lanl.gov
} 
discrete and continuous Fourier transforms. Unlike the Fourier transform, however, wavelets offer users the versatility of selecting from a large family of wavelet transforms based on different filters, allowing the user to optimize the transform for specific applications.

The above-mentioned properties make wavelet transforms well-suited for applications involving non-stationary or aperiodic data, or data that contains transient features or singularities. For instance, we have had great success using DWTs for compressing digital data, such as digitized images and the output of supercomputer simulations. Other research has indicated a wide variety of potential applications for wavelet transforms, including: finiteelement solutions of partial differential equations (PDEs); discretization of integral operators and computational tomography; compression of acoustic data; speech analysis; edge detection and texture analysis; and geophysical signal processing.

\section{Importance to LANL's Science and Technology Base and National R\&D Needs}

Our focus on wavelet transform technology is producing immediate payoffs for both national and Laboratory R\&D needs. Team members are currently helping the FBI develop a nationwide standard for DWT-based compression of digitized fingerprint images as the FBI converts its fingerprint database into a digital format. Our team was chosen for this task ahead of a number of leading academic experts based on our combination of strong theoretical understanding and our ability to produce high-performance software implementations. The standard, which is being developed with FBI funding, incorporates theoretical results on symmetric extension transforms produced by team members in work supported by LDRD funding.

The Multiresolution Seamless Image Database (MrSID), an LDRD project, was developed for the purpose of performing seamless wavelet compression of very large contiguous images in a format compatible with multiresolution browsing. The United States Geological Survey (USGS) Mapping Division has shown an interest in this technology for the distribution of their Digital Ortohophoto Quadrangle (DOQ) data on CD-ROM and over the Internet. A patent application is in preparation for the MrSID technology. Such tools are likely to be useful for other scientific data visualization applications, as well as data compression for high-performance computing environments.

Team members have developed a wavelet image-sequence coding application of potentially great significance to LANL's mission in the arms control arena: remote surveillance and change detection for nuclear materials inventory verification. The same theoretical properties that make wavelet transforms good for coding transient or multiscale phenomena 
also make wavelet coding well-suited for detecting subtle changes in a scene under video surveillance. Team members have demonstrated that the potential exists for combining very high-quality image compression (for archival use in monitoring scenarios) with image-based change detection.

Team members have developed wavelet compression/decompression software for surveillance image sequence data, which has been incorporated into the Experimental Inventory Verification System (EIVS). EIVS will be used by the Department of Energy (DOE) and the International Atomic Energy Agency (IAEA). The EIVS compression algorithm contains an image authentication method that is based on a compression data embedding technology developed with LDRD funding.

As a new piece of basic science, we believe that wavelet transform technology will prove very important in the long run to LANL's science and technology base. Its unique properties satisfy a growing need for a general data analysis tool with the combination of flexibility and low implementation cost lacking in existing data processing methods.

\section{Scientific Approach And Results}

Research in this project has concentrated on topics in multirate filter bank theory relevant to both the design and implementation of wavelet transforms. The goal has been to design and implement so-called filter bank lattice factorizations, architectures that combine optimally low implementation complexity with a flexible framework for application-specific filter design. Team members have produced two new methods to date for designing such lattice structures for 2-channel linear-phase filter banks employed in image coding applications; one of these results was presented at the 1994 IEEE International Symposium on Time/Frequency and Time/Scale Analysis. These new theoretical results have been implemented in software by team members, greatly improving computational performance.

Team members have studied multichannel FIR filter banks with filters satisfying various symmetry conditions. This work has led to a complete classification of all multichannel FIR filter banks in terms of fundamental building blocks that can be used to construct the relevant filter banks. We have also developed computer software to decompose filter banks with symmetries into their fundamental building blocks. This work can be used both to design efficient chips and to accelerate software.

Team members have worked on a new technique known as the embedded zero-tree wavelet transform image compression algorithm. This algorithm has many potential applications in data compression problems involving browsing applications, i.e., situations in which there is a need to view low resolution renderings of images rapidly from some database 
stored in a multi-resolution hierarchy at a remote location. We have studied the use of arithmetic coding in such schemes and have found it to outperform previous Huffman coding methods.

Progress also continues on software implementation of wavelet transform algorithms for use on Lab supercomputers; results were presented at the 1995 IEEE/NASA Data Compression Conference on implementations making use of the $\mathrm{A}++/ \mathrm{P}+$ array class library being developed at Los Alamos. This work has involved the participation of half a dozen graduate research assistants over the past three summers, under the supervision of team members.

In the past year, new theoretical results have been obtained on the characterization of rational transfer matrices with polynomial (FIR) inverses, generalizing and simplifying known results for polynomial transfer matrices with polynomial inverses. These classes are canonical in a certain sense so understanding their intrinsic structure contributes to factorization theory for arbitrary filter banks. This is research at a very abstract level, encompassing general multiinput/multi-output linear systems, and it may have applications outside of filter bank theory; e.g., in convolutional (error-correction) coding.

LDRD funding has also provided for visits to LANL by numerous academic guests, maintaining the team's close ties to academic research in filter bank and wavelet theory. Team members, in turn, have been active in the wavelet research community, giving presentations and invited lectures at such forums as the Supercomputing Expos, the IEEE Data Compression Conferences, the IMACS World Congress on Computation and Applied Math, American Mathematical Society meetings, and the 1995 AAAS annual meeting.

\section{Publications}

1. Bradley, Jon; Brislawn, Chris; and Faber, Vance, "Reflected Boundary Conditions for Multirate Filter Banks," Proc. IEEE Symp. on Time-Frequency and Time-Scale Analysis, 307 (1992).

2. Bradley, Jon; and Brislawn, Chris, "Vector Quantization of Discrete Wavelet Transform Coefficients, CNLS Newsletter, 84, 1 (1992).

3. Bradley, Jon; and Brislawn, Chris, "Wavelet Transform-Vector Quantization Compression of Supercomputer Ocean Models," Proc. IEEE Data Compression Conf., 224 (1993). 
4. Bradley, Jon; and Brislawn, Chris, "Applications of Wavelet-Based Compression to Multidimensional Earth Science Data," Proc. NASA Space Earth Science Data Compression Workshop, 3191, 13 (1993).

5. Bradley, Jon; Brislawn, Chris; Quinlan, Dan; Zhang, H. Davison; and Nuri, Veyis, "Wavelet Subband Coding of Computer Simulation Output Using the A++ Array Class Library," Proc. NASA Space Earth Science Data Compression Workshop, 95-8, 57 (1995).

6. Brislawn, Chris, "Rational Transfer Matrices with FIR Inverses, submitted for publication (1994).

7. Bradley, Jon; and Brislawn, Chris, "SPECTRUM Analysis of Multispectral Imagery in Conjunction with Wavelet/KLT Data Compression," Proc. IEEE Asilomar Conf., 26 (1993).

8. Brislawn, Chris, "Preservation of Subband Symmetry in Multirate Signal Coding," IEEE Transactions on Signal Processing, to be published (1995).

9. Brislawn, Chris, "Classification of Nonexpansive Symmetric Extension Transforms for Multirate Filter Banks," Applied \& Computational Harmonic Analysis, submitted for publication (1994).

10. Brislawn, Chris, "A Simple Lattice Architecture for Even Order Linear Phase Perfect Reconstruction Filter Banks," Proc. IEEE Symp. on Time-Freq. and Time-Scale Analysis, 124 (1994). 